\title{
Ultrafast, Polarized, Single-Photon Emission from m-Plane InGaN Quantum Dots on GaN Nanowires
}

Tim J. Puchtler, ${ }^{* \dagger}{ }^{\dagger}$ Tong Wang, ${ }^{\dagger}$ Christopher X. Ren, ${ }^{\ddagger}$ Fengzai Tang, ${ }^{\ddagger}$ Rachel A. Oliver,

Robert A. Taylor, ${ }^{\dagger}$ and Tongtong $\mathrm{Zhu}^{*,+}$

${ }^{\dagger}$ Department of Physics, University of Oxford, Parks Road, Oxford OX1 3PU, U.K.

${ }^{\ddagger}$ Dept. Materials Science and Metallurgy, University of Cambridge, 27 Charles Babbage Road, Cambridge CB3 0FS, U.K.

\section{Supporting Information}

ABSTRACT: We demonstrate single-photon emission from self-assembled m-plane InGaN quantum dots (QDs) embedded on the side-walls of $\mathrm{GaN}$ nanowires. A combination of electron microscopy, cathodoluminescence, time-resolved microphotoluminescence $(\mu \mathrm{PL})$, and photon autocorrelation experiments give a thorough evaluation of the QD structural and optical properties. The QD exhibits antibunched emission up to $100 \mathrm{~K}$, with a measured autocorrelation function of $g^{(2)}(0)=0.28(0.03)$ at $5 \mathrm{~K}$. Studies on a statistically significant number of QDs show that these m-plane QDs exhibit very fast radiative lifetimes $(260 \pm 55 \mathrm{ps})$ suggesting smaller internal fields than any of the previously reported c-plane and a-plane

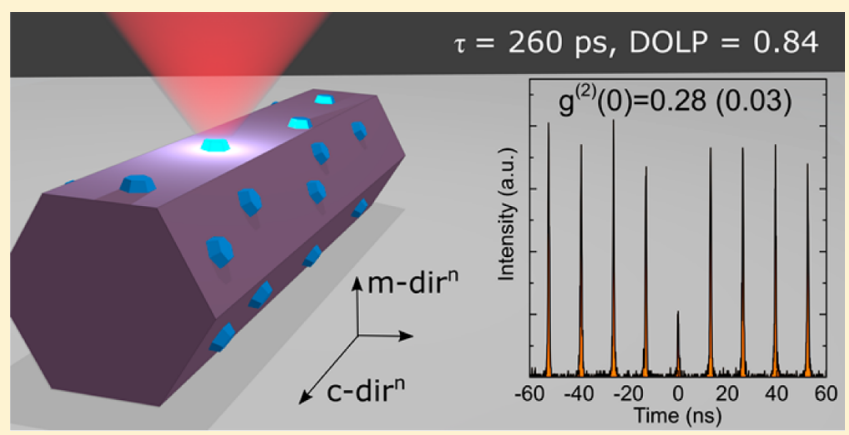
QDs. Moreover, the observed single photons are almost completely linearly polarized aligned perpendicular to the crystallographic $c$-axis with a degree of linear polarization of $0.84 \pm 0.12$. Such InGaN QDs incorporated in a nanowire system meet many of the requirements for implementation into quantum information systems and could potentially open the door to wholly new device concepts.

KEYWORDS: m-Plane, InGaN, quantum dot, single photon, polarized

$\mathrm{T}$ he ability to generate on-demand single photons is of vital importance to quantum information technology such as quantum cryptography, linear optical quantum computing, and quantum metrology. ${ }^{1-3}$ Due to their high stability, good repetition rates, and practicable incorporation into cavities and electronically pumped structures, ${ }^{4}$ quantum dots (QDs) are ideal candidates for the generation of, and interaction with, single photons. Notably nitride QDs offer the advantages of large exciton binding energies and high band offsets, which allow higher temperature operation. ${ }^{5,6}$ InGaN QDs in particular allow access to the blue spectral range ideally suited for freespace communications and efficient fast single-photon detectors. However, due to their wurtzite crystal structure, the orientation of quantum well (QW) and QD nanostructures relative to the crystal axis is of great importance; most work in the nitrides features growth on the polar (0001) c-plane, causing large in-built fields across the heterostructures leading to decreased oscillator strengths of exciton transitions via the quantum-confined Stark effect (QCSE). This limitation on single-photon repetition rates and the unpolarized emission from c-plane QDs constitute major limitations to their application to quantum information systems. Additionally the exciton binding energy in c-plane structures is theoretically lower than that expected in nonpolar orientations. ${ }^{7}$ As such there are significant benefits to be realized if QDs can be fabricated on nonpolar planes.
Recent advances in the growth of InGaN QDs on (11 $\overline{2} 0)$ aplane $\mathrm{GaN}$ have demonstrated some of the advantages associated with the reduced internal fields of nonpolar planes. ${ }^{8,9}$ Additionally the in-plane anisotropy of growth on nonpolar planes causes splitting of the upper valence bands and gives these QDs a large degree of inherent polarization. ${ }^{10}$ This polarized single-photon emission has been shown to persist at temperatures beyond $220 \mathrm{~K},{ }^{11}$ making them candidates for onchip solutions in polarization encoded quantum information protocols. However, fabrication of devices on (1100) m-plane $\mathrm{GaN}$ is challenging due to the lack of well-suited latticematched substrates. ${ }^{12}$ While efficient m-plane QW devices have proven difficult to achieve, requiring expensive free-standing $\mathrm{GaN}$ substrates to avoid high defect densities, they have shown better emission properties than even a-plane QWs, having faster radiative recombination times, higher optical gain, and an increased degree of linear polarization, ${ }^{13-16}$ although the exact reasons for this are not fully understood. However, there has been minimal success in the growth of QDs on the m-plane perhaps because the indium incorporation efficiency is much lower in the m-plane than the c-plane and as such traditional

Received: September 23, 2016

Revised: November 23, 2016

Published: November 29, 2016 

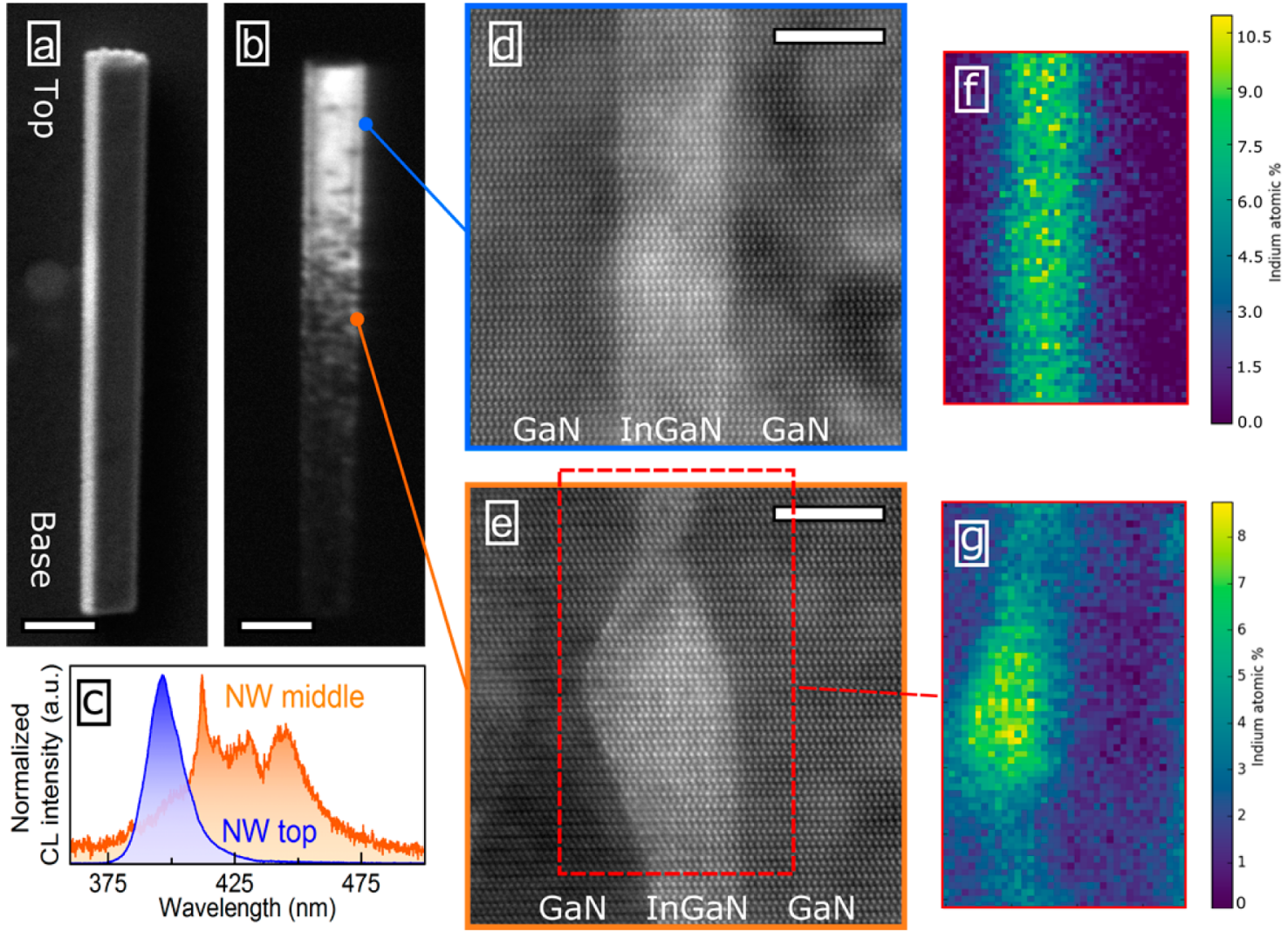

Figure 1. (a) SEM image of a NW with (b) corresponding CL image showing a transition from fractured to uniform InGaN emission toward the top of the NW. Scale bar represents $1 \mu \mathrm{m}$. (c) Representative CL emission spectra for these two regions, showing QW-like emission toward the top of the NW and sharper peaks within the fractured region. Corresponding TEM images of the InGaN layers (d) at the top and (e) from the middle of the NW showing QW- and QD-like morphologies, respectively. Scale bar represents $3 \mathrm{~nm}$. The indium distribution may be easily seen in corresponding EDX spectra, showing (f) a consistent width of the InGaN layer toward the top of the NW and ( $\mathrm{g}$ ) small clusters of indium in the middle of the NW.

QD growth methods have not translated well onto m-plane substrates. ${ }^{17}$ While claims have been made for fabrication of $\mathrm{m}$ plane QDs, ${ }^{18-20}$ only the formation of nanostructures on $\mathrm{m}$ plane facets has been demonstrated; there is currently no single-QD spectroscopy that shows optical emission from individual nanostructures, provides evidence of their QD-like behavior, or demonstrates their superior properties relative to c-plane QDs.

Here, we present a novel self-assembled growth method for an m-plane QD system with corresponding structural and optical characterization and the first direct proof of the quantum nature of emission in such a system. By the use of nanowires (NWs), we allow the relaxation of in-plane strain leading to a reduction in defects and an increase in optical extraction efficiencies from the ends of the NWs. The reduction of internal fields and the QCSE has already been demonstrated in such a geometry using QW emitters. ${ }^{21}$ In the future, the position of the QDs near the outer surface of the NWs may allow coupling of the QDs into the photonic modes of the NW, as described by $\mathrm{Yu}$ et al. ${ }^{22}$ The structure consists of a selfcatalytic GaN NW core grown by metal-organic vapor phase epitaxy (MOVPE). We intentionally supply a substantial $\mathrm{SiH}_{4}$ flux during the GaN NW growth step to (i) greatly enhance the vertical growth rate and (ii) act as an antisurfactant that will roughen the NW sidewalls and lead to the formation of threedimensional nanostructures during the growth of 5 InGaN/ GaN QW layers, which are deposited in a core-shell configuration achieving QD formation. Details of the sample growth are given in the Experimental Methods section. Formation of m-plane QDs on the sidewalls of hexagonal NWs occurs with both the NW and QD growth performed in a single uninterrupted MOVPE run without the requirement of a previously defined mask, thereby reducing complexity and fabrication time. Such m-plane QD structures can efficiently generate ultrafast linearly polarized single photons up to $100 \mathrm{~K}$, which can potentially be coupled into the photonic modes of the hexagonal nanowires.

Structural properties of the NWs have been assessed using scanning electron microscopy (SEM) with an attached cathodoluminescence detector (SEM-CL) allowing emission spectra to be collected with high spatial resolution from regions excited locally by the electron beam. Variation in emission along the NW can be seen in Figure la,b. We see that the InGaN emission at the base and middle of the NW consists of many sharp peaks attributed to separate localization centers. Emission becomes stronger and more uniform toward the NW tip, displaying QW-like emission centered at $400 \mathrm{~nm}$, having minimal spectral overlap with the sharp emission features observed.

To gain further insight into the InGaN layer morphology, high-resolution transmission electron microscopy (TEM) imaging has been performed on cross-sectional lamella prepared by focused ion beam (FIB) milling. It reveals that the distribution of indium is of a uniform thickness toward the top of the NWs (Figure 1d), consistent with the SEM-CL spectra showing QW-like emission. However, throughout most 
of the NW, the InGaN layers appear nonuniform, with variations in both thickness and concentration. Energydispersive X-ray (EDX) spectroscopy performed concurrently shows the presence of high indium-content structures (approximately $5 \mathrm{~nm}$ high $\times 15 \mathrm{~nm}$ across in size) throughout these nonuniform layers (Figure $1 \mathrm{~g}$ ), which when combined with the SEM-CL spectra showing isolated sharp peaks confirms the formation of QDs.

A silicon flux during growth has been shown to greatly enhance the vertical growth rate of GaN NWs. Tessarek et al. suggested that during this process a $\mathrm{SiN}_{x}$ layer or highly $\mathrm{Si}$ doped $\mathrm{GaN}$ surface layer can be incorporated and formed on the m-plane sidewalls of the NWs due to (1) the presence of $\mathrm{Ga}$ droplets on top of the GaN NWs during growth, which attract the Ga atoms, and (2) the low solubility of $\mathrm{Si}$ and $\mathrm{N}$ in liquid Ga. ${ }^{23}$ In the growth of our NWs, we supplied a constant $\mathrm{SiH}_{4}$ flux $(0.2 \mu \mathrm{mol} / \mathrm{min})$ during the $\mathrm{GaN} N W$ growth in order to increase the vertical growth rate. This $\mathrm{SiH}_{4}$ was stopped prior to the InGaN QW growth, to encourage the formation of a core-shell structure. The carrier gas was then changed to $\mathrm{N}_{2}$, and the growth temperature was ramped down to the QW growth temperature with a constant supply of ammonia. We suggest that this process leads to a variation of $\mathrm{SiN}_{x}$ coverage along the nanowire length, with more $\mathrm{SiN}_{x}$ at the bottom of the nanowire, which had been exposed to $\mathrm{SiH}_{4}$ for a longer time. The formation of a $\mathrm{SiN}_{x}$ layer on planar surfaces is known to lead to a transition from $2 \mathrm{D}$ to $3 \mathrm{D}$ growth. ${ }^{24}$ This so-called "antisurfactant effect" of Si on a NW's surface is postulated to disturb the growth of the GaN beneath the first InGaN QW layer (perhaps by the formation of 3D islands, which then partially coalesce). This results in a rougher surface toward the bottom of the NW for the subsequent $\mathrm{InGaN} / \mathrm{GaN}$ QWs to grow on. Higher InGaN growth rate (thickness), and higher local indium incorporation (composition) due to the presence of nanoscale facets as confirmed by the TEM and EDX analysis (Figure 1e,g), ${ }^{25}$ lead to the formation of m-plane QD-like structures on the NW's sidewalls. Additional structural information and discussion of sample growth is presented in the Supporting Information (SI). And a more detailed structural and chemical analysis of the effect of $\mathrm{SiN}_{x}$ on InGaN/GaN core-shell NW growth will be presented elsewhere.

Optical emission characteristics of the NWs were measured using microphotoluminescence $(\mu \mathrm{PL})$ under two-photon excitation $(\lambda=800 \mathrm{~nm})$, as this has been shown to give a relatively larger absorption cross-section for objects of increased quantum confinement and hence increases $\mathrm{QD}$ signal relative to the nonuniform QW background. ${ }^{26}$ Consistent, smooth QW-like emission typically centered at $415 \pm 2 \mathrm{~nm}$ is observed from the top of the NW, while sharp emission peaks are observed at other positions along the NW (Figure 2a), showing strong similarities to the emission observed under SEM-CL. Several of these peaks are present in each NW (average of 3.6 QDs per NW, standard deviation 3.1, for $50 \mathrm{NWs}$ examined) with a relatively wide distribution of emission wavelengths (average $473 \mathrm{~nm}$, standard deviation $\sigma=28 \mathrm{~nm}$, for 66 peaks examined).

Power dependent measurements have been performed to allow identification of any biexcitons present. The power dependence of the $\mathrm{QD}$ emission varies approximately with the square of the excitation power $\left(\alpha P^{2.1}\right.$, see Figure $2 \mathrm{a}$, inset) as expected for an exciton created via a two-photon absorption process, with only two biexcitons observed having power
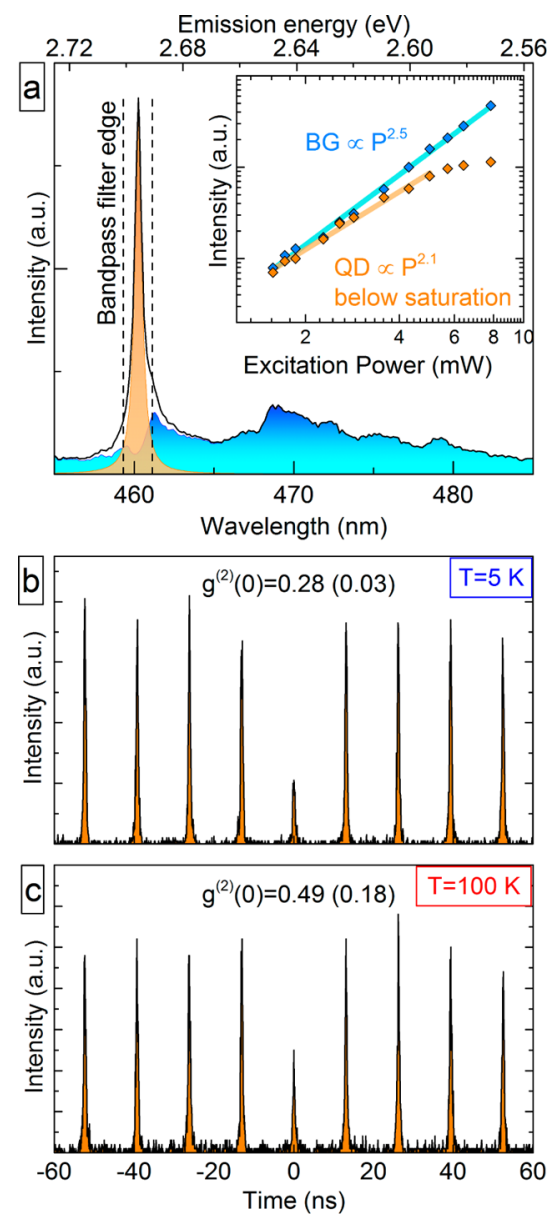

Figure 2. (a) Example emission spectrum from a QD at $5 \mathrm{~K}$ showing a single peak attributed to a $\mathrm{QD}$ and a wide background emission attributed to fractured QW in its vicinity. Example fits of the QD, background, and filters used for background correction in autocorrelation measurements are shown. (Inset) Emission intensity follows a near-quadratic power dependence until saturation, as expected for a two-photon absorption process. Autocorrelation measurements of a QD at (b) $5 \mathrm{~K}$ showing significant antibunching of $g^{(2)}(0)=0.28$ and $(c) 100 \mathrm{~K}$ showing antibunching behavior with $g^{(2)}(0)=0.49$.

dependence to the exponent 4.4. The rarity of observed biexcitons may suggest a large biexciton binding energy or simply a weak peak that is hidden by the background emission at higher powers beyond the saturation of the exciton peak ( 6 $\mathrm{mW}$ in Figure 2). These peaks cannot clearly be attributed to individual exciton peaks, so no estimate of the biexciton binding energy is made. The emission wavelength appears relatively insensitive to excitation power with no clear correlation observable for studied QDs. While some degree of spectral diffusion is observed (typically peak wavelengths vary by $\sim 0.05$ $\mathrm{nm}$ over a period of tens of seconds), which may be concealing a power dependent emission shift, the small magnitude strongly suggests that the in-built fields, which are screened at higher excitation power densities for c-plane QDs, are minimal in the QDs currently observed.

In order to prove that the emission features come from individual quantum emitters, autocorrelation measurements have been performed using a Hanbury Brown and Twiss setup. QD emission is spectrally filtered using a pair of tunable bandpass filters, although some degree of background emission still 
remains (illustrated in Figure 2a). While the background emission cannot be completely removed by spectral filtering, clear antibunching is observed in the second-order autocorrelation function (Figure $2 \mathrm{~b}$ ) giving a correlation signal at zero time delay of $g^{(2)}(0)=0.28$. This value can be corrected by removing the effect of the uncorrelated background emission according to $\left(g_{\text {cor }}^{(2)}(0)-1\right) \rho^{2}=\left(g_{\text {raw }}^{(2)}(0)-1\right)$ where $\rho$ is the ratio of $\mathrm{QD}$ signal to total intensity measured. ${ }^{27}$ Doing so gives a value of $g_{\text {cor }}^{(2)}(0)=0.03$. This nonzero value may be explained by re-excitation of the QD from background emission during a single excitation pulse but is more likely caused by inaccurate estimations of $\rho$ : difficulties arise as the tunable filter's transmission is not a perfect boxcar function, having a small but significant transmission beyond the intended transmission window. Errors may also arise in the corrected value if the QD peak shape is incorrectly fitted, such as the influence of broadening mechanisms or phonon sidebands, which may become significant at higher temperatures. (See SI for information on estimations of $\rho$.) This is evident in additional autocorrelation measurements performed at $100 \mathrm{~K}$, which give values of $g_{\mathrm{rar}}^{(2)}(0)$ of 0.49 and $g_{\text {cor }}^{(2)}(0)$ of 0.18 (Figure $2 \mathrm{c}$ ). While the value of $\rho$ becomes lower and more difficult to estimate accurately as the QD thermally broadens, antibunching behavior remains at this significantly elevated temperature. We should also note that the sample was grown with $5 \mathrm{InGaN}$ layers in order to increase the likelihood of QD formation. However, given that most nanowires contained several measurable QDs, a reduction in background emission can be achieved by simply reducing the number of layers present.

While the formation of QDs has been seen in TEM and sharp peaks have been observed in $\mu \mathrm{PL}$, one might consider the possibility that the two are not directly correlated; it is possible that the emission peaks are related to excitons bound to defects or impurities present in the NWs, an especially important consideration given the role of high silicon levels in the formation mechanism of the QDs themselves. However, first we should note that the excitation power near saturation does not vary with the square-root of excitation density and as such suggests that the emission is not defect-related. ${ }^{28}$ In order to confirm this assessment, temperature dependent $\mu \mathrm{PL}$ measurements have been performed on a representative emission peak, as the emission peaks from QDs and defects behave differently with temperature. Example spectra for a single emission peak from 4-220 K may be seen in Figure 3 with the emission wavelength increasing smoothly, with a rate increasing with temperature as expected for semiconductor band-gaps following the Varshni equation. ${ }^{29}$ Alternatively, for defect related emission, we would expect to observe a progression in peak intensity representing the transition from bound to free excitons as we increasingly thermalize the donor-bound excitons. ${ }^{30}$ Indeed the wavelength change fits almost perfectly $\left(R^{2}=0.996\right)$ to the O'Donnell-Chen model of temperature dependence of band gaps (see SI for details), and as such we can conclude that the emission features presented do correspond to semiconductor localization centers such as those observed in TEM.

The variation of line width with increasing temperature gives us insight into the influence of phonons on these m-plane QDs. We expect significant acoustic-phonon broadening in the nitrides, ${ }^{31}$ while having minimal coupling to optical phonons at the range of temperatures measured due to the large optical phonon energy in $\mathrm{GaN}(92 \mathrm{meV}){ }^{32}$ The line width $\Gamma$ of QDs fits well with the standard equation for bulk or QW

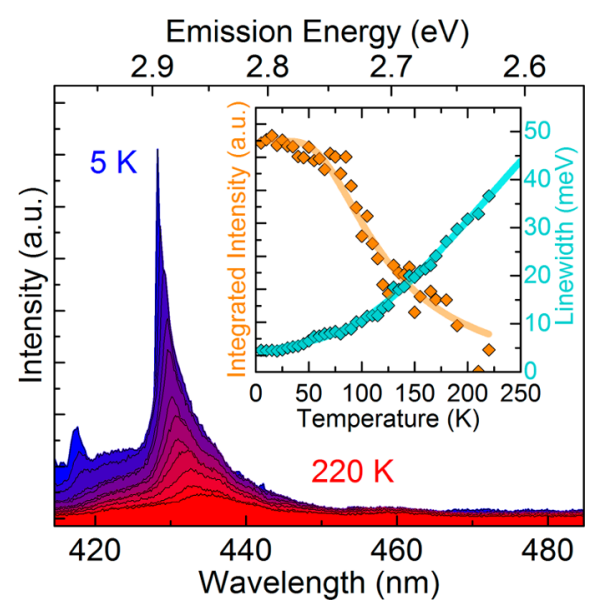

Figure 3. Variation in $\mathrm{QD}$ emission with temperature up to $220 \mathrm{~K}$, showing a gradual red-shift and (inset) decrease in intensity and increase in line width with increasing temperature as expected for QD emission rather than defect-related emission.

semiconductors, ${ }^{33} \Gamma(T)=\Gamma_{0}+\gamma_{\mathrm{a}} T+\gamma_{\mathrm{b}} \exp \left(-E_{\mathrm{a}} /\left(k_{\mathrm{B}} T\right)\right)$ with terms representing the zero-phonon line width (ZP-line width), acoustic phonon coupling, and delocalization of carriers from the QD, respectively. We can see in Figure 3 (inset) that the acoustic phonon coupling is indeed the most significant for temperatures up to $\sim 100 \mathrm{~K}$, giving a coupling constant of $50 \pm$ $7 \mu \mathrm{eV} / \mathrm{K}$ and $\Gamma_{0}$ of $3.7 \pm 0.4 \mathrm{meV}$. These line widths are several times higher than many other reported values for InGaN QDs. ${ }^{31,33,34}$ We have attributed the larger-than-expected ZPline width to the likely significant presence of spectral diffusion in the sample, given the probable high density of point defects associated with the $\mathrm{Si}$ dopants, which act as charge trapping sites in the regions in which QDs are formed, as discussed previously. It would seem consistent that we observe a correspondingly larger acoustic phonon coupling constant, as the magnitude of the spectral diffusion is also expected to increase with temperature. ${ }^{35}$ Therefore, this value of $\gamma_{a}$ is not purely the acoustic coupling but contains a measure of the increased thermal movement of carriers as well.

The reduction in integrated intensity from the QD has been plotted in Figure 3 (inset) and fits well with an Arrhenius-type equation based on a single carrier escape energy, $I=I_{0} /\left(1+A_{1}\right.$ $\left.\exp \left(-E_{1} /\left(k_{\mathrm{B}} T\right)\right)\right)$. The energy $E_{1}$ representing the energy required for an exciton to escape the QD localization is extracted as $35 \pm 1 \mathrm{meV}$. This corresponds excellently to the extracted value of the localization depth used in fitting the line width previously, which yielded $E_{\mathrm{a}}=38 \pm 2 \mathrm{meV}$, with these values being slightly larger than those previously reported in literature for c-plane InGaN QDs, and hence explaining the higher operational temperature we have observed compared to those studies. 33,36

Note that due to the fast lifetimes, the homogeneous line width of the QDs is beyond our resolution limit. Hence to extract fwhm and amplitude data for Figure 3, we have used Gaussian functions to fit our emission peaks, representing inhomogeneous broadening mechanisms such as spectral diffusion.

To gain insights into the exciton recombination dynamics of the QDs, time-resolved measurements have been performed on 32 spectrally isolated QDs. Excitation power was chosen to be well below saturation for each QD to reduce any effect of carrier-generated internal fields. Decay traces fit well with two- 

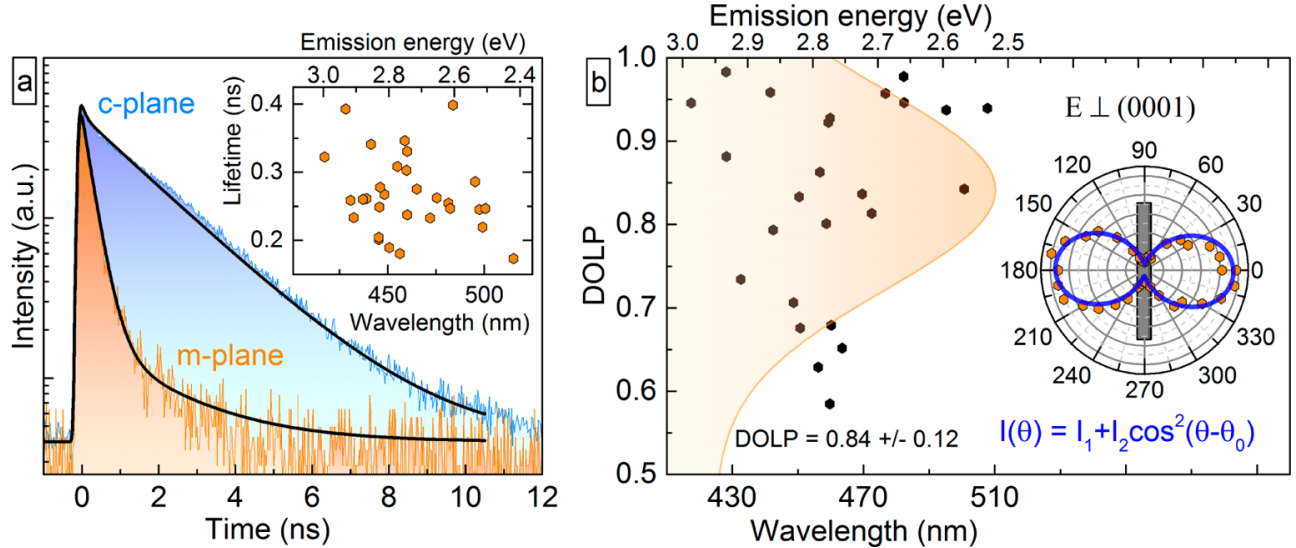

Figure 4. (a) Time-resolved PL decay traces of the m-plane QDs studied compared to the longer lifetime peaks sometimes present when exciting the tip of the NW attributed to c-plane QDs. Average lifetimes are $260 \pm 55$ ps, suggesting minimal internal fields within the QDs, which show (inset) no correlation to emission wavelength. (b) Degree of linear polarization measurements against emission wavelength, showing a high average DOLP of 0.84 with no wavelength dependence. The shaded Gaussian is a guide for the eye representing the distribution of DOLPs measured. (Inset) An example $360^{\circ}$ polarization plot showing the orientation of polarization relative to the NW, with all QDs measured exhibiting polarization orthogonal to the NW growth direction. Data collected at $5 \mathrm{~K}$.

component exponential decays, with one strong fast component attributed to the QD and a significantly weaker (by orders of magnitude) slower component. As such, and noting the QD is weakly pumped while the emission is spatially and spectrally isolated, the stretching parameter often used to account for temporally varying lifetimes or ensembles of emitters (the Kohlrausch function) is not required. ${ }^{37}$ To allow measurement of very fast lifetimes, fitting of the biexponential decay is convolved with the detector's Gaussian instrument response function (IRF, $\sim 150 \mathrm{ps}$ width). An example may be seen in Figure 4a. The lifetimes are mostly insensitive to changes in temperature (see SI), although a slight reduction at higher temperatures is sometimes observed, which may show the onset of thermally activated nonradiative recombination.

The lifetime of the QD exciton was found to be as low as 170 ps, with an average of $260 \pm 55$ ps for the 32 QDs measured. This value is much lower than typical lifetimes for c-plane QDs (typically $1-10 \mathrm{~ns})^{38,39}$ supporting the assertion that the nonpolar orientation of these QDs reduces the effect of their built-in fields, reducing the QCSE and increasing oscillator strength. Interestingly these lifetimes are also significantly faster than those reported in InGaN QDs grown on the a-plane, typically of $\sim 500 \mathrm{ps}{ }^{8,9}$ suggesting that these QDs better avoid residual in-built fields, which may be caused by any semipolar facets, than do their a-plane counterparts. Indeed, these lifetimes are less than those observed in quantum disks, which have claimed to be free from internal fields $(\sim 300 \mathrm{ps}) .^{40}$

The lack of significant fields within the QDs is further supported, albeit tentatively, by the lack of wavelength dependence of the lifetime of the excitons; typically exciton binding energy decreases with increasing QD size, decreasing emission energy. ${ }^{41}$ Similarly we expect an increase in emission lifetime as the electron-hole wave function overlap is reduced, with a magnitude dependent on the internal field and geometry of the QD. The resulting inverse correlation of emission energy to lifetime strongly depends on the presence of built-in fields, ${ }^{37}$ assuming isolated transitions are measured. While our QD growth relies on a stochastic formation process, which results in a range of $\mathrm{QD}$ sizes and compositions, we see no such relationship for our QDs (Figure 4a, inset, gives an $R^{2}$ of 0.02 to a linear fit).
We should note that for several NWs, QD emission may be observed with much longer lifetimes ( $2 \mathrm{~ns})$ exclusively when excited at their top end. We believe these may indicate the presence of c-plane QDs formed by fluctuations in the c-plane QW on the top surface of the NW, although detailed analysis of the c-plane structure at the top of the NW has not been performed.

Finally, we investigate the polarization properties of the QDs. In m-plane heterostructures, we expect a high degree of inplane polarization, even without the presence of anisotropic inplane strain or in-built fields, as the crystallographic asymmetry lifts the degeneracy of the top two p-like valence band states. $^{42,43} \mathrm{~A}$ high degree of polarization has already been repeatedly demonstrated in m-plane QW structures. ${ }^{44,45}$ However, it is possible that shape anisotropy of these QDs could randomize the polarization via the nonequal strain fields affecting the $\mathrm{A}$ and $\mathrm{B}$ valence band states differently. ${ }^{46,47}$

An example of the QD intensity as a function of polarization angle is presented in Figure $4 \mathrm{~b}$ (inset), fitted with $I(\theta)=I_{1}+I_{2}$ $\cos ^{2}\left(\theta-\theta_{0}\right)$ with $\theta_{0}$ as the angle of polarization and $I_{1}$ and $I_{2}$ as the minimum and maximum intensities, respectively. Polarization measurements performed on 26 QDs are presented in Figure $4 \mathrm{~b}$ and show a high degree of linear polarization, defined as DOLP $=\left(I_{2}-I_{1}\right) /\left(I_{1}+I_{2}\right)$, measured at $0.84 \pm 0.12$. For all QDs measured, the angle of polarization is aligned orthogonal to the NWs, hence in the $\mathrm{E} \perp \mathrm{c}$ direction as expected from $\mathrm{m}$ plane QWs. We also note that the DOLP has no dependence on the wavelength of $\mathrm{QD}$ emission (a linear fit gives an $R^{2}$ of 0.03 ). We can therefore conclude that the polarization is robust to any shape anisotropy present in these QDs.

In conclusion, we have presented the first successful growth of m-plane QDs using a novel method in which the QDs selfassemble owing to higher InGaN growth rate and indium content on the nanofacets induced by $\mathrm{SiN}_{x}$ on the sidewalls of hexagonal GaN NWs and have demonstrated their antibunched emission. We have performed detailed analysis on the optical properties of these QDs, and we conclude that they show very fast lifetimes (as low as 170 ps) suggesting the presence of only minimal internal fields. The QDs also show a high degree of linear polarization $(D O L P \approx 0.84)$ and antibunching $g^{(2)}(0)=$ $0.28(0.03)$, although the QDs also exhibit a high level of 
spectral diffusion likely caused by higher impurities in the material on which the QDs form.

Experimental Methods. Sample Growth. The NWs were grown by metal-organic vapor phase epitaxy in a $6 \times 2$ in. Thomas Swan close-coupled showerhead reactor on 2 in. cplane sapphire substrates using trimethylgallium, trimethylindium, and ammonia as precursors, hydrogen as carrier gas, and $\mathrm{SiH}_{4}$ as source of silicon. After annealing the sapphire substrate at $1050{ }^{\circ} \mathrm{C}$, a low temperature nucleation $\mathrm{GaN}$ layer $(\sim 5 \mathrm{~nm})$ was deposited at $550{ }^{\circ} \mathrm{C}$ and annealed in $\mathrm{H}_{2}$ at $1000{ }^{\circ} \mathrm{C}$ to act as a mask for the subsequent NW growth. The NWs were then grown at $1000{ }^{\circ} \mathrm{C}$ with a reactor pressure of 300 Torr and a V/ III ratio of 50, during which a constant $\mathrm{SiH}_{4}$ flow of $(0.2 \mu \mathrm{mol} /$ min) was supplied to enhance the vertical growth rate. Fiveperiod core-shell InGaN/GaN multiple quantum wells were grown on the GaN core NWs using a V/III ratio of 5000 and $\mathrm{N}_{2}$ as the carrier gas, whereby the $\mathrm{InGaN}$ was grown at $730{ }^{\circ} \mathrm{C}$ and the $\mathrm{GaN}$ barriers were grown at $850^{\circ} \mathrm{C}$. Samples have been harvested and redeposited on a silicon substrate for analysis.

Characterization. Cathodoluminescence measurements were performed at $30 \mathrm{~K}$ using a Philips XL30 SEM operating at $3 \mathrm{kV}$, which was equipped with a Gatan MonoCL4. Sitespecific TEM (transmission electron microscopy) samples were prepared from NWs using the dual beam FIB-SEM (focused ion beam-scanning electron microscopy) based lift-out technique (FEI Helios NanoLab). Atomic resolution HAADF (high-angle annular dark field)-STEM images were taken using a spherical aberration-corrected TEM (FEI $\left.\operatorname{Titan}^{3} 80-300\right)$ at $300 \mathrm{kV}$ with screen currents between 50 and $250 \mathrm{pA}$, whereas chemical composition analysis was performed by an analytical TEM (FEI Tecnai Osiris) fitted with four EDX spectrometers operating at $200 \mathrm{kV}$. TEM analysis was carried out with electron beam parallel to the $11 \overline{2} 0$ direction. $\mu \mathrm{PL}$ measurements were performed using a mode-locked Ti:sapphire laser emitting at $800 \mathrm{~nm}(1.5$ ps pulse length, $76 \mathrm{MHz}$ repetition rate) through a $0.7 \mathrm{NA}$ objective (spot size $\sim 0.6 \mu \mathrm{m}^{2}$ ) with the emission passing through the same objective before being passed to a $0.5 \mathrm{~m}$ spectrometer with a $1200 \mathrm{lpmm}$ grating (maximal resolution of $0.04 \mathrm{~nm}$ ). The sample is held in a closed-cycle cryostat (AttoDRY800). Lifetime measurements were performed passing a wavelength selected output from the spectrometer to a photomultiplier tube (PMT, instrument response function width $\sim 150 \mathrm{ps}$ ) connected to a timecorrelated single-photon counting module (binning resolution 25 ps) triggered by the Ti:sapphire pulses. Autocorrelation measurements were performed passing the QD signal through a pair of tunable bandpass filters (Semrock Versachrome) to a pair of PMTs through a 50:50 beamsplitter in a Hanbury Brown and Twiss configuration. Polarization measurements were performed using a half-wave plate and linear polarizer in front of the spectrometer.

\section{ASSOCIATED CONTENT}

\section{S Supporting Information}

The Supporting Information is available free of charge on the ACS Publications website at DOI: 10.1021/acs.nanolett.6b03980.

QD formation, signal to background estimations, emission energy vs temperature, and temperature dependence of lifetimes and polarization (PDF)

\section{AUTHOR INFORMATION}

\section{Corresponding Authors}

*Tim J. Puchtler. E-mail: tim.puchtler@physics.ox.ac.uk. Tel: +44 1865272390 .

*Tongtong Zhu. E-mail: tz234@cam.ac.uk.

ORCID

Tim J. Puchtler: 0000-0002-3215-2960

Tong Wang: 0000-0001-9530-2965

\section{Author Contributions}

The experiments were conceived by T.J.P and T.Z., optical measurements were performed by T.J.P. and T.W., SEM-CL measurements were performed by T.Z., samples were grown by T.Z., TEM sample preparation and imaging were performed by C.X.R. and F.T., data analysis was performed and the paper was written by T.J.P., growth and structural analysis was supervised by R.A.O., and optical characterization was supervised by R.A.T.

\section{Funding}

This research was supported by the Engineering and Physical Sciences Research Council (EPSRC) UK (Grant Nos. EP/ M012379/1 and EP/M011682/1). The microscopy studies were supported by the European Research Council under the European Community's Seventh Framework Programme (FP7/2007-2013)/ERC grant agreement no. 279361 (MACONS).

\section{Notes}

The authors declare no competing financial interest.

Data presented in the figures of this article is available at http://dx.doi.org/10.5287/bodleian:54OrJnNXw.

\section{REFERENCES}

(1) Beveratos, A.; Brouri, R.; Gacoin, T.; Villing, A.; Poizat, J.-P.; Grangier, P. Phys. Rev. Lett. 2002, 89 (18), 187901.

(2) Knill, E.; Milburn, G. J.; Laflamme, R. Nature 2001, 409, 46-52.

(3) Giovannetti, V.; Lloyd, S.; Maccone, L. Science 2004, 306, 13301336.

(4) Buckley, S.; Rivoire, K.; Vučković, J. Rep. Prog. Phys. 2012, 75 (12), 126503.

(5) Monemar, B. Phys. Rev. B 1974, 10 (2), 676-681.

(6) Holmes, M. J.; Choi, K.; Kako, S.; Arita, M.; Arakawa, Y. Nano Lett. 2014, 14 (2), 982-986.

(7) Funato, M.; Kawakami, Y. J. Appl. Phys. 2008, 103 (9), 093501.

(8) Griffiths, J. T.; Zhu, T.; Oehler, F.; Emery, R. M.; Fu, W. Y.; Reid, B. P. L.; Taylor, R. A.; Kappers, M. J.; Humphreys, C. J.; Oliver, R. A. APL Mater. 2014, 2 (12), 126101.

(9) Zhu, T.; Oehler, F.; Reid, B. P. L.; Emery, R. M.; Taylor, R. A.; Kappers, M. J.; Oliver, R. A. Appl. Phys. Lett. 2013, 102, 251905.

(10) Reid, B. P. L.; Kocher, C.; Zhu, T.; Oehler, F.; Chan, C. C. S.; Oliver, R. A.; Taylor, R. A. Appl. Phys. Lett. 2015, 106 (17), 171108.

(11) Wang, T.; Puchtler, T. J.; Zhu, T.; Jarman, J. C.; Nuttall, L. P.; Oliver, R. A.; Taylor, R. A. https://arxiv.org/abs/1610.00152.

(12) Ren, C. X. Mater. Sci. Technol. 2016, 32 (5), 418-433.

(13) Park, S.-H.; Ahn, D.; Chuang, S.-L. IEEE J. Quantum Electron. 2007, 43 (12), 1175-1182.

(14) Lee, J.; Ni, X.; Wu, M.; Li, X.; Shimada, R.; Özgür, Ü.; Baski, A. A.; Morkoç, H.; Paskova, T.; Mulholland, G.; Evans, K. R. Appl. Phys. Lett. 2009, 95, 101106.

(15) Zhang, S.; Cui, Y.; Griffiths, J. T.; Fu, W. Y.; Freysoldt, C.; Neugebauer, J.; Humphreys, C. J.; Oliver, R. A. Phys. Rev. B: Condens. Matter Mater. Phys. 2015, 92, 245202.

(16) Marcinkevicius, S.; Kelchner, K. M.; Nakamura, S.; Denbaars, S. P.; Speck, J. S. Phys. Status Solidi Curr. Top. Solid State Phys. 2014, 11 (3-4), 690-693. 
(17) Yamada, H.; Iso, K.; Saito, M.; Masui, H.; Fujito, K.; DenBaars, S. P.; Nakamura, S. Appl. Phys. Express 2008, 1, 041101.

(18) Yoo, H.-I.; Ra, Y.-H.; Navamathavan, R.; Choi, Y.-H.; Park, J.H.; Lee, C.-R. CrystEngComm 2014, 16, 7580-7586.

(19) Yang, X.; Arita, M.; Kako, S.; Arakawa, Y. Appl. Phys. Lett. 2011, 99 (6), 061914.

(20) Yang, X.; Arita, M.; Kako, S.; Arakawa, Y. Phys. Status Solidi Curr. Top. Solid State Phys. 2012, 9 (3-4), 613-615.

(21) Koester, R.; Hwang, J. S.; Salomon, D.; Chen, X.; Bougerol, C.; Barnes, J. P.; Dang, D. L. S.; Rigutti, L.; De Luna Bugallo, A.; Jacopin, G.; Tchernycheva, M.; Durand, C.; Eymery, J. Nano Lett. 2011, 11 (11), 4839-4845.

(22) Yu, Y.; Dou, X. M.; Wei, B.; Zha, G. W.; Shang, X. J.; Wang, L.; Su, D.; Xu, J. X.; Wang, H. Y.; Ni, H. Q.; Sun, B. Q.; Ji, Y.; Han, X. D.; Niu, Z. C. Adv. Mater. 2014, 26 (17), 2710-2717.

(23) Tessarek, C.; Heilmann, M.; Butzen, E.; Haab, A.; Hardtdegen, H.; Dieker, C.; Spiecker, E.; Christiansen, S. Cryst. Growth Des. 2014, 14 (3), 1486-1492.

(24) Markurt, T.; Lymperakis, L.; Neugebauer, J.; Drechsel, P.; Stauss, P.; Schulz, T.; Remmele, T.; Grillo, V.; Rotunno, E.; Albrecht, M. Phys. Rev. Lett. 2013, 110 (3), 036103.

(25) Tang, F.; Barnard, J. S.; Zhu, T.; Oehler, F.; Kappers, M. J.; Oliver, R. A. Appl. Phys. Lett. 2015, 107 (8), 082104.

(26) Collins, D.; Jarjour, A. F.; Hadjipanayi, M.; Taylor, R. A.; Oliver, R. A.; Kappers, M.; Humphreys, C.; Tahraoui, A. Nanotechnology 2009, 20, 245702.

(27) Brouri, R.; Beveratos, A.; Poizat, J. P.; Grangier, P. Opt. Lett. 2000, 25 (17), 1294-1296.

(28) Reshchikov, M. A.; Morkoç, H. J. Appl. Phys. 2005, 97 (6), 061301.

(29) Varshni, Y. P. Physica 1967, 34 (1), 149-154.

(30) Belloeil, M.; Gayral, B.; Daudin, B. Nano Lett. 2016, 16 (2), 960-966.

(31) Demangeot, F.; Simeonov, D.; Dussaigne, A.; Butté, R.; Grandjean, N. Phys. Status Solidi 2009, 6, S598-S601.

(32) Barker, A. S.; Ilegems, M. Phys. Rev. B 1973, 7 (2), 743-750.

(33) Sebald, K.; Lohmeyer, H.; Gutowski, J.; Yamaguchi, T.; Hommel, D. Phys. Status Solidi C 2006, 3 (11), 3864-3868.

(34) Oliver, R. A.; Briggs, G. A. D.; Kappers, M. J.; Humphreys, C. J.; Yasin, S.; Rice, J. H.; Smith, J. D.; Taylor, R. A. Appl. Phys. Lett. 2003, 83 (4), 755-757.

(35) Empedocles, S. A.; Bawendi, M. G. J. Phys. Chem. B 1999, 103 (11), 1826-1830.

(36) Seguin, R.; Rodt, S.; Strittmatter, A.; Reissmann, L.; Bartel, T.; Hoffmann, A.; Bimberg, D.; Hahn, E.; Gerthsen, D. Appl. Phys. Lett. 2004, 84 (20), 4023-4025.

(37) Winkelnkemper, M.; Dworzak, M.; Pereira, T. S.; Bartel, T. Unpublished work, 2007.

(38) Robinson, J. W.; Rice, J. H.; Jarjour, A. F.; Smith, J. D.; Taylor, R. A.; Oliver, R. A.; Briggs, G. A. D.; Kappers, M. J.; Humphreys, C. J.; Arakawa, Y. Appl. Phys. Lett. 2003, 83 (13), 2674.

(39) Winkelnkemper, M.; Dworzak, M.; Bartel, T. P.; Strittmatter, A.; Hoffmann, A.; Bimberg, D. Phys. Status Solidi B 2008, 245 (12), 2766-2770.

(40) Bardoux, R.; Kaneta, A.; Funato, M.; Kawakami, Y.; Kikuchi, A.; Kishino, K. Phys. Rev. B: Condens. Matter Mater. Phys. 2009, 79 (15), 155307.

(41) Elward, J. M.; Chakraborty, A. J. Chem. Theory Comput. 2013, 9

(10), 4351-4359.

(42) Ghosh, S.; Waltereit, P.; Brandt, O.; Grahn, H.; Ploog, K. Phys.

Rev. B: Condens. Matter Mater. Phys. 2002, 65 (7), 075202.

(43) Schulz, S.; Tanner, D. P.; O’Reilly, E. P.; Caro, M. A.; Martin, T. L.; Bagot, P. A. J.; Moody, M. P.; Tang, F.; Griffiths, J. T.; Oehler, F.; Kappers, M. J.; Oliver, R. A.; Humphreys, C. J.; Sutherland, D.; Davies, M. J.; Dawson, P. Phys. Rev. B: Condens. Matter Mater. Phys. 2015, 92 (23), 235419

(44) Sun, Y. J.; Brandt, O.; Ramsteiner, M.; Grahn, H. T.; Ploog, K. H. Appl. Phys. Lett. 2003, 82 (22), 3850-3852.
(45) Liuolia, V.; Marcinkevicius, S.; Lin, Y.-D.; Ohta, H.; Denbaars, S. P.; Nakamura, S. J. Appl. Phys. 2010, 108 (2), 023101.

(46) Winkelnkemper, M.; Seguin, R.; Rodt, S.; Schliwa, A.; Reissmann, L.; Strittmatter, A.; Hoffmann, A.; Bimberg, D. J. Appl. Phys. 2007, 101 (11), 113708.

(47) Teng, C.-H.; Zhang, L.; Hill, T. A.; Demory, B.; Deng, H.; Ku, P.-C. Appl. Phys. Lett. 2015, 107 (19), 191105. 